San Jose State University

SJSU ScholarWorks

Faculty Publications

Physics and Astronomy

$1-1-2011$

\title{
The fossil record of two-phase galaxy assembly: Kinematics and metallicities in the nearest $\mathrm{SO}$ galaxy
}

\author{
J, A, Arnold \\ UCO/Lick Observatory
}

Aaron J. Romanowsky

San Jose State University, aaron.romanowsky@sjsu.edu

J. P. Brodie

UCO/Lick Observatory

L. Chomiuk

Harvard-Smithsonian Center for Astrophysics

L. R. Spitler

Swinburne University

See next page for additional authors

Follow this and additional works at: https://scholarworks.sjsu.edu/physics_astron_pub

Part of the Astrophysics and Astronomy Commons

\section{Recommended Citation}

J, A, Arnold; Aaron J. Romanowsky; J. P. Brodie; L. Chomiuk; L. R. Spitler; J. Strader; A. J. Benson; and D. A. Forbes. "The fossil record of two-phase galaxy assembly: Kinematics and metallicities in the nearest S0 galaxy" Astrophysical Journal Letters (2011). https://doi.org/10.1088/2041-8205/736/2/L26

This Article is brought to you for free and open access by the Physics and Astronomy at SJSU ScholarWorks. It has been accepted for inclusion in Faculty Publications by an authorized administrator of SJSU ScholarWorks. For more information, please contact scholarworks@sjsu.edu. 


\section{Authors}

J, A, Arnold; Aaron J. Romanowsky; J. P. Brodie; L. Chomiuk; L. R. Spitler; J. Strader; A. J. Benson; and D. A. Forbes 


\title{
THE FOSSIL RECORD OF TWO-PHASE GALAXY ASSEMBLY: KINEMATICS AND METALLICITIES IN THE NEAREST SO GALAXY
}

\author{
Jacob A. Arnold ${ }^{1}$, Aaron J. Romanowsky ${ }^{1}$, Jean P. Brodie ${ }^{1}$, Laura Chomiuk $^{2,3}$, Lee R. Spitler ${ }^{4}$, Jay Strader $^{2}$, \\ ANDREW J. Benson ${ }^{5}$, AND DunCAN A. FORBES 4 \\ ${ }^{1}$ UCO/Lick Observatory, University of California, Santa Cruz, CA 95064, USA \\ ${ }^{2}$ Harvard-Smithsonian Center for Astrophysics, 60 Garden St., Cambridge, MA 02138, USA \\ ${ }^{3}$ National Radio Astronomy Observatory, P.O. Box O, Socorro, NM 87801, USA \\ ${ }^{4}$ Centre for Astrophysics \& Supercomputing, Swinburne University, Hawthorn, VIC 3122, Australia \\ ${ }^{5}$ California Institute of Technology, MS 105-24, 1200 East California Boulevard, Pasadena, CA 91125, USA \\ Received 2011 January 29; accepted 2011 May 27; published 2011 July 7
}

\begin{abstract}
We present a global analysis of kinematics and metallicity in the nearest S0 galaxy, NGC 3115, along with implications for its assembly history. The data include high-quality wide-field imaging from Suprime-Cam on the Subaru telescope, and multi-slit spectra of the field stars and globular clusters (GCs) obtained using KeckDEIMOS/LRIS and Magellan-IMACS. Within two effective radii, the bulge (as traced by the stars and metal-rich $\mathrm{GCs})$ is flattened and rotates rapidly $(v / \sigma \gtrsim 1.5)$. At larger radii, the rotation declines dramatically to $v / \sigma \sim 0.7$, but remains well aligned with the inner regions. The radial decrease in characteristic metallicity of both the metalrich and metal-poor GC subpopulations produces strong gradients with power-law slopes of $-0.17 \pm 0.04$ and $-0.38 \pm 0.06 \mathrm{dex} \mathrm{dex}^{-1}$, respectively. We argue that this pattern is not naturally explained by a binary major merger, but instead by a two-phase assembly process where the inner regions have formed in an early violent, dissipative phase, followed by the protracted growth of the outer parts via minor mergers with typical mass ratios of $\sim 15-20: 1$.
\end{abstract}

Key words: galaxies: bulges - galaxies: formation - galaxies: halos - galaxies: individual (NGC 3115) - galaxies: kinematics and dynamics - globular clusters: general

Online-only material: color figures

\section{INTRODUCTION}

The faint outer regions of galaxies are particularly useful probes of the earliest phases of galaxy formation. Here relaxation times are long, and subtle formational clues are preserved not only in their integrated stellar light but also in bright tracer populations such as globular clusters (GCs) and planetary nebulae $(\mathrm{PNe})$.

Recently, wide field observations of integrated stellar light and PNe (Proctor et al. 2009; Coccato et al. 2009) have revealed that the relative kinematic homogeneity among the central regions of early-type galaxies (ETGs; Emsellem et al. 2007) gives way to surprising diversity in the outlying parts, e.g., central fast rotators can sometimes rotate slowly at larger radii.

The implication is that inner and outer regions are somewhat decoupled. In the classic gas-rich binary major-merger scenario for forming galaxy bulges, the spatial dependence of dissipational processes could produce dramatic kinematical transitions in radius (Hoffman et al. 2010).

Alternatively, inner and outer spheroids may form in two separate phases, which could explain the strong size and internal density evolution of ETGs from $z \sim 2$ to today (e.g., Daddi et al. 2005; van Dokkum et al. 2008). This inside-out growth hypothesis means that the inner regions form early as compact stellar spheroids while the outer parts grow later through the accretion of smaller galaxies (e.g., Naab et al. 2009; Oser et al. 2010).

One way to distinguish between the merger and inside-out scenarios is to search for their signatures in the properties of GC systems. In their archetypal study, Searle \& Zinn (1978) used the joint distribution of spatial, kinematical, age, and metallicity properties of GCs to infer the hierarchical buildup of the Milky
Way's outer halo. A key concept here is that building galaxies via the stochastic infall of satellites leads to flattened metallicity gradients at large radius, an effect now observed within the GC systems of two ETGs (Harris 2009; Forbes et al. 2011; cf also Coccato et al. 2010). Likewise, in wet major merger remnants gas dissipation should create metallicity gradients in the central regions, with flatter profiles in the outer regions where any initial gradients have been mixed up in the merger (e.g., White 1980; Hopkins et al. 2009). The similar expectations for large-scale metallicity structure from each of these scenarios necessitates additional information, such as from kinematics, to place such results in the proper context.

A useful complication with the GCs is their bimodality, as each galaxy generally has a metal-poor and a metal-rich subpopulation (MPGCs and MRGCs). In the Milky Way these are associated with the stellar halo and the bulge or thick disk, respectively, while in ETGs there are stronger MRGC components reflecting their more dominant bulges. These subpopulations permit the investigation of two distinct phases of galaxy assembly (stellar halo and bulge) far beyond the Local Group.

As part of our ongoing surveys of ETGs we have studied the nearest giant S0 galaxy, NGC 3115 (distance $9 \mathrm{Mpc}$, bulge effective radius $R_{e}=57^{\prime \prime}=2.5 \mathrm{kpc}, B / T \geqslant 0.9$, inclination $86^{\circ}$; Capaccioli et al. 1987), in unprecedented photometric and spectroscopic detail. In particular, we have obtained extensive kinematic data sets of its field stars and GCs. Here we map out the global kinematic and metallicity structure of its dominant, extended bulge, and examine some implications for its assembly history.

In Section 2, we describe our imaging and spectroscopic observations. Kinematic and metallicity profiles are presented in Section 3. We discuss how these results constrain the formation 


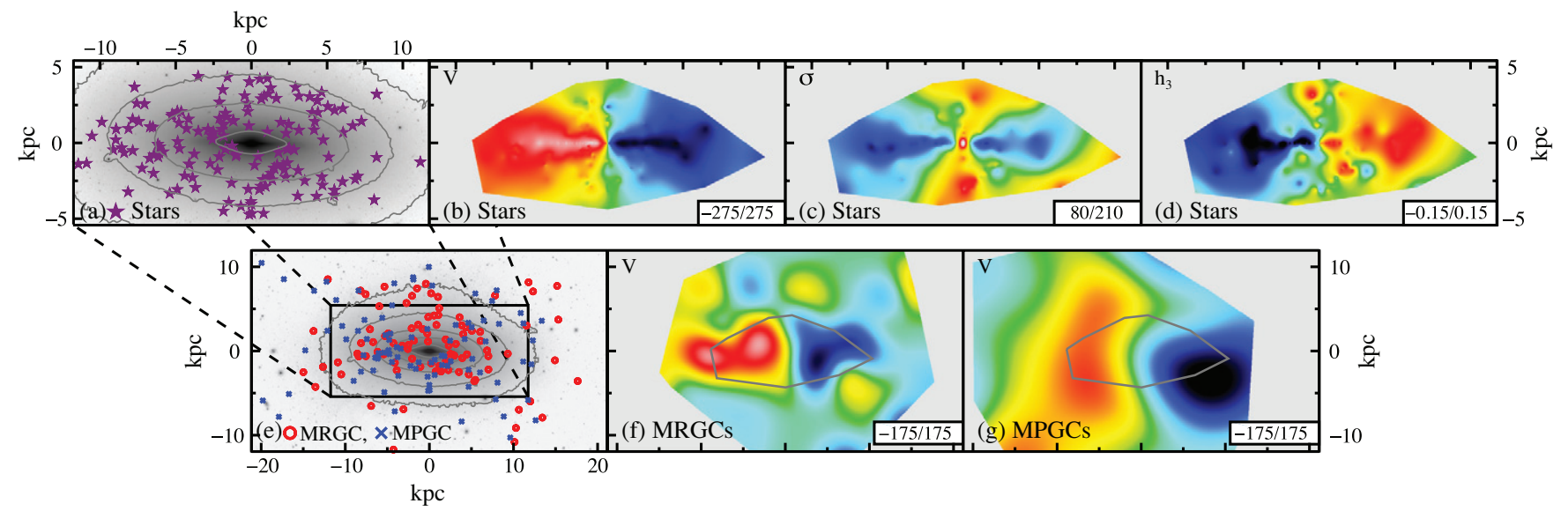

Figure 1. Spatial and kinematic maps of NGC 3115. Top: $i$-band image (a) with SKiMS (stellar light) measurement locations (purple stars). Maps of stellar rotation (b), velocity dispersion (c), and $h_{3}$ (d), each sharing the same spatial scale as (a). Bottom: zoomed-out view (e) with spectroscopically confirmed metal-rich (red circles) and metal-poor GC (blue crosses) locations. The inset rectangle denotes the region shown in (a) where selected $i$-band isophotes are also overplotted. Rotation maps, made using the MRGC (f) and MPGC (g) data sets, with the same spatial scale as (e), and overlaid by an outline of the zoomed-in stellar fields from (b)-(d). The kinematic maps were created using kriging techniques for statistically reconstructing sparsely sampled functions, using "interpolation" and "fitting" approaches for the stellar and GC data sets, respectively (Press et al. 2007). The minimum/maximum color-bar scaling is recorded in the bottom right corner of each panel (all in units of $\mathrm{km} \mathrm{s}^{-1}$ except for (d), which is dimensionless). While these maps effectively convey the broad-brush kinematic behavior, we note that interpolating sparse data sets may lead to oversmoothing and anomalous small-scale structure, particularly at larger radius.

(A color version of this figure is available in the online journal.)

mechanisms of the outer bulge and halo in Section 4, and summarize the conclusions in Section 5.

\section{OBSERVATIONS}

Here, we briefly summarize the observations used in the present analysis; complete details will be presented in a followup paper. We obtained gri images with 0.'5-0.'7 seeing using Suprime-Cam on the $8.2 \mathrm{~m}$ Subaru telescope. Sections of this imaging are shown in Figures 1(a) and (e) to provide orientation for the spectroscopic measurements discussed below. The photometry allows us to select GC candidates for follow-up spectroscopy and to characterize the distributions of number density and metallicity for the GC system. Color is used as a proxy for metallicity since GCs are generally old (see Brodie \& Strader 2006; Kuntschner et al. 2002). The color boundary we use for MPGCs/MRGCs in NGC 3115 is $(g-i)_{0}=0.91 \mathrm{mag}$ $([\mathrm{Fe} / \mathrm{H}]=-0.8 \mathrm{dex})$.

We performed spectroscopy using DEIMOS on the Keck-II telescope to focus on the calcium triplet region ( $\sim 8580 \AA, R \sim 5200$; see Romanowsky et al. 2009; Foster et al. 2011). We have confirmed 150 GCs and also obtained spectra of the background galaxy light in 166 slits for use in a novel technique called "SKiMS" (Stellar Kinematics from Multiple Slits; Proctor et al. 2009; Foster et al. 2011). Another 15 and 11 unique GC spectra were obtained using LRIS ( 3500-7500 A, $R \sim 1100-2200)$ and IMACS $(\sim 4000-7000 \AA, R \sim 2200)$ on the Keck-I and Magellan telescopes, respectively.

GC velocities were measured by cross-correlating their absorption-line spectra with stellar templates. We imposed a lower velocity cut at $350 \mathrm{~km} \mathrm{~s}^{-1}$ to remove foreground stars. Fainter SKiMS spectra were co-added to reach a target $\mathrm{S} / \mathrm{N}$ of 25 using the Voronoi 2D-binning method by Cappellari \& Copin (2003). The stellar line-of-sight velocity distribution of each spectrum was parameterized as a truncated Gauss-Hermite series $\left(V, \sigma, h_{3}, h_{4}\right)$ using pPXF (Cappellari \& Emsellem 2004) and 13 template stars, with parameter uncertainties estimated using Monte Carlo modeling of data sets with added noise.
We supplement our SKiMS catalog with long-slit stellar kinematics data from Norris et al. (2006) and Illingworth \& Schechter (1982), and incorporate 20 GC velocities from previous studies (Kuntschner et al. 2002; Puzia et al. 2004).

\section{RESULTS}

Figure 1 shows two-dimensional maps of the kinematic data. In the top row, the thin disk is evident as a narrow stripe within an extended and rapidly rotating bulge. The thin disk's lowered velocity dispersion, and anti-correlated $V$ and $h_{3}$, extend well into the bulge, suggesting an additional embedded component like a very thick disk. On larger scales (Figure 1, bottom row), distinct rotation is seen in the MRGC and MPGC subsystems, while there is a general trend for the rotation to weaken in the outer regions.

To simplify the rotation and dispersion trends into onedimensional profiles, we use a variation of the "kinemetry" technique developed for the SAURON survey (Krajnović et al. 2006) as optimized for data with discrete spatial and velocity sampling (Proctor et al. 2009; Foster et al. 2011). This method samples the kinematic field (e.g., rotation) using concentric elliptical annuli, and fits the data to flattened sinusoidal models as a function of position angle. For the discrete velocity data (GCs), rotation and dispersion are fitted simultaneously through a maximum likelihood method (Figures 2(a)- (d)). The position angles and ellipticities of the rotation field and the sampling bins (P.A.kin, $\epsilon_{\text {kin }}$ ) are part of the fit for the SKiMS data but are not well constrained for the discrete velocity data, which we assume follows the stellar isophotes (P.A. $=43.5, \epsilon=0.5$ ). Our results are insensitive to reasonable variations in these parameters. Uncertainties are estimated via Monte Carlo fitting of mock data sets.

The resulting rotation profiles for the different subcomponents are shown in Figures 2(e) and (f), where rolling fits with radius are used to capture the details of any radial kinematic transitions (e.g., from the inner to outer bulge/halo). Within $\sim 1.5 R_{\mathrm{e}}$ the MRGC system rotates nearly as rapidly as the stellar bulge, supporting the coevolution of these two components, 

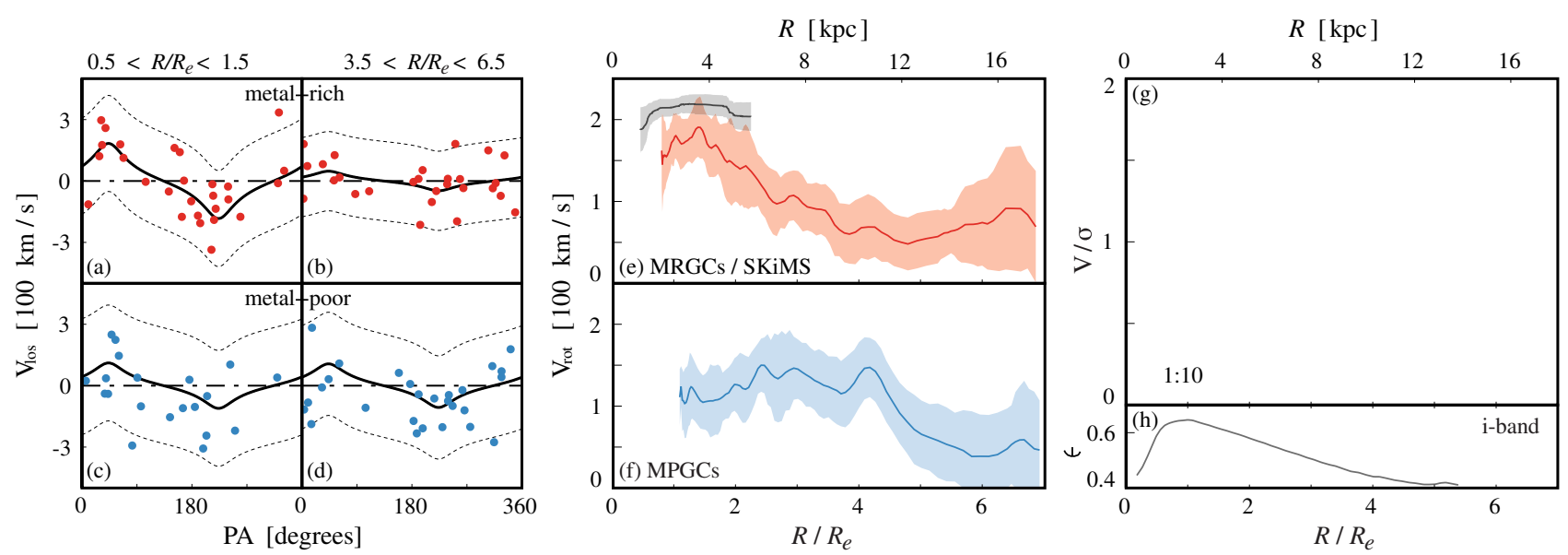

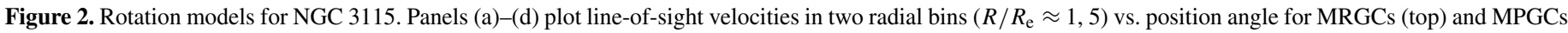

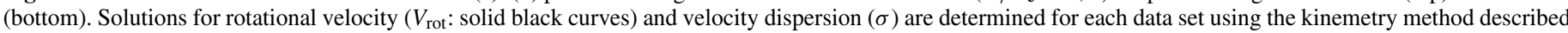

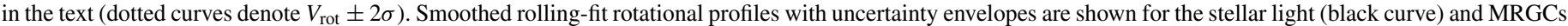

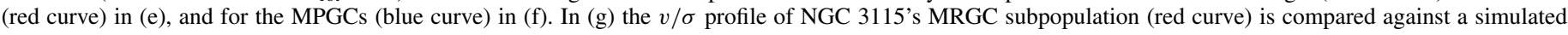
merger remnant with a 1:10 mass ratio (dashed: Bournaud et al. 2005). (h) The $i$-band ellipticity profile.

(A color version of this figure is available in the online journal.)
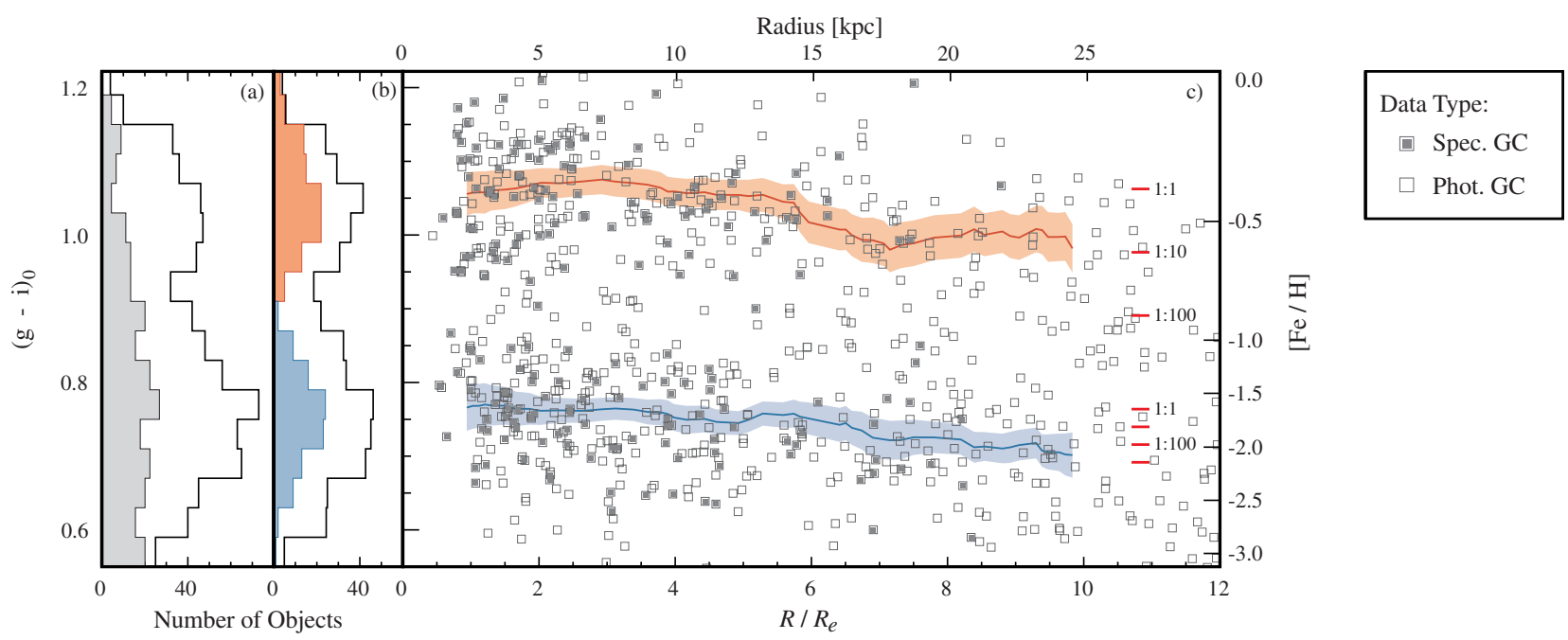

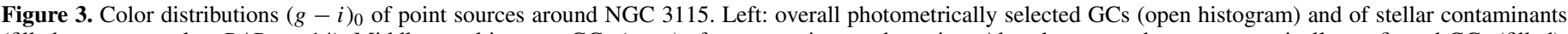

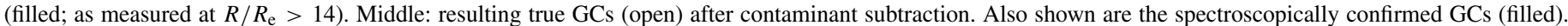

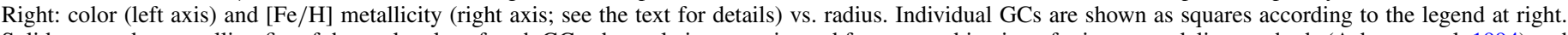

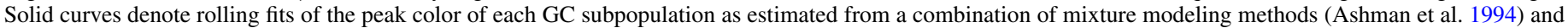

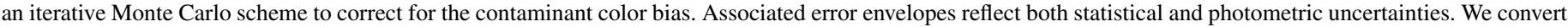

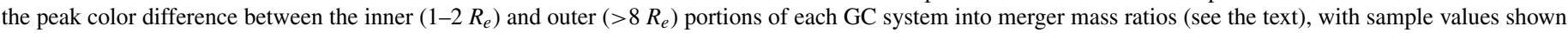
on the right-hand side.

(A color version of this figure is available in the online journal.)

as also inferred from their similar ages and metallicities (Norris et al. 2006). At larger radii, this rotation decreases dramatically (see also Figures 2(a) and (b)). The MPGCs have moderate rotation with a decline outside $\sim 4 R_{\mathrm{e}}$.

An alternative rotation profile for the MRGCs is shown in Figure 2(g), after normalizing by the local velocity dispersion. The photometric ellipticity profile is also plotted, showing a decrease with radius that parallels the rotational gradient. The overall implication is for a bulge that has a high degree of rotational flattening in its central regions, while becoming rounder and dispersion-dominated in its outskirts.

Having found kinematic transitions in both GC subpopulations, we look for analogous transitions in the radial metallicity profiles. First we summarize the overall color distribution of the GCs in Figure 3(a), which shows a classic bimodality. We will assume that this bimodality persists with increas- ing radius, but that the location of the color peaks may shift. At large radii we must cope with the contaminating effects of foreground stars, whose color distribution we also show in Figure 3(a), and use to construct Monte Carlo mock data sets to iteratively correct for the contaminant bias on the color peak locations.

Figure 3(c) shows color versus radius, both for individual GC candidates and for the fitted peak locations. Both GC subpopulations have radially decreasing colors, which we quantify as power-law color gradients with slopes of -0.05 and $-0.07 \mathrm{mag} \mathrm{dex}^{-1}$ for MPGCs and MRGCs, respectively. Using our own empirical calibration to the $(g-z)$ color used in Advanced Camera for Surveys GC studies (Peng et al. 2006), the gradients are -0.07 and $-0.10 \mathrm{mag} \mathrm{dex}^{-1}$. Converting to $[\mathrm{Fe} / \mathrm{H}]$ metallicity (Peng et al. 2006), we estimate gradients of $-0.38 \pm 0.06$ and $-0.17 \pm 0.04 \mathrm{dex} \mathrm{dex}^{-1}$. 
To our knowledge, this is the first time that metallicity gradients in both GC subpopulations have been measured to large radii in any galaxy besides a few very massive ellipticals (see Section 1). It is also one of the first cases of any galaxy type where joint rotation and metallicity gradients are observed in the halo (see also NGC 4697: Méndez et al. 2005, 2009; and NGC 4125: Pu et al. 2010).

\section{IMPLICATIONS}

We now consider some possible implications of the rotation and metallicity gradients for NGC 3115's assembly history. The central bulge properties are generally consistent with a standard major merger picture, with the very high amount of rotation in this case indicative of a gas-rich merger with an uneven mass ratio (e.g., Bournaud et al. 2005; Naab et al. 2006). Alternatively, the inner bulge might have formed via the inward migration of giant star-forming clumps within a turbulent disk fed by cold streams from the cosmic web at early epochs (e.g., Noguchi 1999; Elmegreen et al. 2008; Dekel et al. 2009). In either case, the exceptionally high inner-bulge rotation in NGC 3115 may require a residual thick disk component (cf. Cretton et al. 2001; Wuyts et al. 2010). Also, the survival of an old thin disk with a mean age of 5-8 Gyr (Norris et al. 2006) means that most of the action in the central regions occurred before $z \sim 0.5-1$, and that there have been no major mergers more recently.

At larger radii there are generic expectations from major mergers for rotation profile behavior. These remnants are generally expected to have rapid outer rotation resulting from both residual disk spin and the conversion of orbital into internal angular momentum (e.g., Bendo \& Barnes 2000; Cretton et al. 2001), in stark contrast to the declining rotation observed in NGC 3115. To make this difference more explicit, we searched through the literature for extended rotation profiles from simulations of major merger remnants, choosing a representative example that comes close to reproducing the central rotation of NGC 3115's bulge and MRGC system. The chosen remnant is the result of a 1:10 spiral-spiral merger from Bournaud et al. (2005), which we overplot in Figure 2(g), showing the discrepancy in the outer regions between the high rotation predicted and the low rotation observed.

Gas-rich 1:1 merger remnants with small pericenters can also produce declining outer rotation, but the outer "dry" part of the remnant is generally expected to show kinematical misalignment with the inner regions (Hoffman et al. 2010; L. Hoffman et al. 2011, in preparation). Possible examples of this scenario include NGC 5128 (Peng et al. 2004) and NGC 4125 (Pu et al. 2010), but other cases like NGC 3115 with decreasing but well-aligned rotation suggest that there must be another explanation (cf. NGC 821 and NGC 3377: Proctor et al. 2009; Coccato et al. 2009; and early arguments along these lines by Scorza \& Bender 1995).

Without exhaustive simulations of major mergers, we cannot rule out the possibility that finely tuned parameters (viewing angle, impact parameter, etc.) would reproduce the observed kinematics of such systems. Nonetheless, it seems more natural to consider a two-phase assembly scenario (Section 1) in which ETGs form inside-out. In this case, inner bulges form at high redshift while subsequent outer bulge and halo growth is driven primarily by dry minor-merger accretion events. The satellites fall in from many different directions and provide little net rotational support (Vitvitska et al. 2002; Abadi et al. 2006; Bournaud et al. 2007; Qu et al. 2010). The radial decline in rotation of the MRGC system could then represent a transition from an inner bulge formed in violent, dissipative processes at high redshift, to an outer spheroid (around one-third of the bulge mass in the case of NGC 3115) built largely from accreted material over a more protracted period.

The MPGCs also show a marked rotational decrease, albeit at a larger radius. Here the theoretical picture is less clear, but we postulate an inner metal-poor stellar halo formed in situ at high $z$ followed by the accretion of outer material that also creates the outer bulge (cf. Zolotov et al. 2009).

Declining metallicity profiles are expected in this two-phase assembly scenario since the lower-mass accreted systems should be more metal-poor than the central galaxy (Naab et al. 2009; Bezanson et al. 2009). The prediction (see Section 1) is of a downward transition from an inner metallicity profile, whose shape reflects the detailed in situ star formation history, to an outer profile flattened by radial mixing and primarily composed of accreted material. We assume that this holds for both the stellar and GC metallicity profiles, though systematic offsets may arise from the difference between the ensemble properties of stars and GCs within incoming satellites (e.g., GCs are typically at larger galactocentric radii). As there are not yet any quantitative predictions, we adopt a schematic model where GC peak metallicities (or colors) are markers of their host galaxies' masses (or luminosities), using the known correlations between these parameters at low $z$ (see below). The peak GC color in the outer bulge/halo then indicates the characteristic luminosity of the accreted systems.

The color-mass relations may well evolve with time, so we take a more general approach of considering the GC color difference between the central and outlying regions as an indicator of the characteristic mass ratio that assembled the outer galaxy. Using the color-mass relations from Peng et al. (2006) for the MPGCs and MRGCs separately, we then generate predicted outer GC peak colors for various stellar mass-ratios 1: $x$ in NGC 3115, overplotting these in Figure 3(c). For the MPGCs we find $x=200(15<x<2900$; the color-mass relation is shallow so this constraint is weak) and for the MRGCs we find $x=8(3<x<19)$. In combination, we estimate $x \sim 15-20$.

Very roughly, the accreted galaxies had luminosities of $\sim 5 \times 10^{8} L_{B, \odot}$, equivalent to a dwarf elliptical. Such galaxies today each host $\sim 20$ MPGCs and $\sim 3$ MRGCs on average (Peng et al. 2008), and could therefore account for a total of $\sim 350$ MPGCs and $\sim 50$ MRGCs in the outer regions of NGC 3115. These numbers are different from the $\sim 260$ and $\sim 140$ GCs that we estimate NGC 3115 to have outside $2 R_{\mathrm{e}}$, but this discrepancy is not significant given the uncertainties in these calculations. A fundamental complication arises from biased galaxy assembly (Brodie \& Strader 2006), where the low-mass galaxies that were accreted at high $z$ are thought to have hosted GCs of higher metallicity than their present-day counterparts in lower density environments. This means that the galaxies accreted by NGC 3115 may have had somewhat lower masses than in our simplified calculation.

As a demonstration of the ongoing accretion process, NGC 3115 does have a dwarf companion of this luminosity at $45 \mathrm{kpc}$ projected distance, NGC 3115B. This galaxy hosts 40 GCs (Kundu \& Whitmore 2001) and we estimate the dynamical friction timescale before it disrupts and adds its stars and GCs to the main galaxy to be $\sim 2$ Gyr.

\section{CONCLUSIONS}

We have found radially decreasing profiles of rotation and metallicity in the GC system of NGC 3115, and compared 
these to theoretical expectations. While the central regions were probably formed in violent, dissipative circumstances such as a gas-rich major merger, this event should have produced high or misaligned outer rotation. A more likely scenario for explaining the assembly of the outer bulge and halo is via dry minor mergers and accretion events, whose mass ratios we have tentatively quantified via the GC metallicities.

Other assembly processes that may contribute to spheroid formation, such as monolithic collapse or quasar-induced expansion (e.g., Fan et al. 2008), have not been considered here and merit future study. Cosmological simulations exploring two-phase assembly predict that up to $80 \%$ of the stellar mass present in today's ETGs has been accreted (Oser et al. 2010). Consequently, kinematical and chemical transitions such as those observed here should be ubiquitous.

We thank Andi Burkert, Avishai Dekel, Loren Hoffman, Chris Moody, and Joel Primack for useful discussions, the referee Igor Chilingarian for helpful comments, and Ewan O'Sullivan for contributing observing time. Based in part on data collected at Subaru Telescope (operated by the National Astronomical Observatory of Japan) via a Gemini Observatory time exchange (GN-2008A-C-12), and also the $6.5 \mathrm{~m}$ Magellan Telescopes located at Las Campanas Observatory, Chile. Some of the data presented herein were obtained at the W. M. Keck Observatory, operated as a scientific partnership among the California Institute of Technology, the University of California and the National Aeronautics and Space Administration, and made possible by the generous financial support of the W. M. Keck Foundation. This material is based upon work supported by the National Science Foundation under Grants AST-0808099 and AST-0909237 and a Graduate Research Fellowship, and also by the UCSC University Affiliated Research Center's Aligned Research Program. A.J.R. was further supported by the FONDAP Center for Astrophysics CONICYT 15010003. A.J.B. acknowledges the support of the Gordon and Betty Moore Foundation. L.C. is a Jansky Fellow at the National Radio Astronomy Observatory. The National Radio Astronomy Observatory is a facility of the National Science Foundation operated under cooperative agreement by Associated Universities, Inc. We acknowledge financial support from the Access to Major Research Facilities Programme, a component of the International Science Linkages Programme established under the Australian Government's innovation statement, Backing Australia's Ability.

\section{REFERENCES}

Abadi, M. G., Navarro, J. F., \& Steinmetz, M. 2006, MNRAS, 365, 747 Ashman, K. M., Bird, C. M., \& Zepf, S. E. 1994, AJ, 108, 2348 Bendo, G. J., \& Barnes, J. E. 2000, MNRAS, 316, 315
Bezanson, R., van Dokkum, P. G., Tal, T., Marchesini, D., Kriek, M., Franx, M. \& Coppi, P. 2009, ApJ, 697, 1290

Bournaud, F., Jog, C. J., \& Combes, F. 2005, A\&A, 437, 69

Bournaud, F., Jog, C. J., \& Combes, F. 2007, A\&A, 476, 1179

Brodie, J. P., \& Strader, J. 2006, ARA\&A, 44, 193

Capaccioli, M., Held, E. V., \& Nieto, J. 1987, AJ, 94, 1519

Cappellari, M., \& Copin, Y. 2003, MNRAS, 342, 345

Cappellari, M., \& Emsellem, E. 2004, PASP, 116, 138

Coccato, L., Gerhard, O., \& Arnaboldi, M. 2010, MNRAS, 407, L26

Coccato, L., et al. 2009, MNRAS, 394, 1249

Cretton, N., Naab, T., Rix, H., \& Burkert, A. 2001, ApJ, 554, 291

Daddi, E., et al. 2005, ApJ, 626, 680

Dekel, A., Sari, R., \& Ceverino, D. 2009, ApJ, 703, 785

Elmegreen, B. G., Bournaud, F., \& Elmegreen, D. M. 2008, ApJ, 688, 67

Emsellem, E., et al. 2007, MNRAS, 379, 401

Fan, L., Lapi, A., De Zotti, G., \& Danese, L. 2008, ApJ, 689, L101

Forbes, D. A., Spitler, L. R., Strader, J., Romanowsky, A. J., Brodie, J. P., \& Foster, C. 2011, MNRAS, 413, 2943

Foster, C., et al. 2011, MNRAS, in press (arXiv:1104.5503)

Harris, W. E. 2009, ApJ, 703, 939

Hoffman, L., Cox, T. J., Dutta, S., \& Hernquist, L. 2010, ApJ, 723, 818

Hopkins, P. F., Cox, T. J., Dutta, S. N., Hernquist, L., Kormendy, J., \& Lauer, T. R. 2009, ApJS, 181, 135

Illingworth, G., \& Schechter, P. L. 1982, ApJ, 256, 481

Krajnović, D., Cappellari, M., de Zeeuw, P. T., \& Copin, Y. 2006, MNRAS, 366,787

Kundu, A., \& Whitmore, B. C. 2001, AJ, 122, 1251

Kuntschner, H., Ziegler, B. L., Sharples, R. M., Worthey, G., \& Fricke, K. J. 2002, A\&A, 395, 761

Méndez, R. H., Teodorescu, A. M., Kudritzki, R., \& Burkert, A. 2009, ApJ, 691,228

Méndez, R. H., Thomas, D., Saglia, R. P., Maraston, C., Kudritzki, R. P., \& Bender, R. 2005, ApJ, 627, 767

Naab, T., Jesseit, R., \& Burkert, A. 2006, MNRAS, 372, 839

Naab, T., Johansson, P. H., \& Ostriker, J. P. 2009, ApJ, 699, L178

Noguchi, M. 1999, ApJ, 514, 77

Norris, M. A., Sharples, R. M., \& Kuntschner, H. 2006, MNRAS, 367, 815

Oser, L., Ostriker, J. P., Naab, T., Johansson, P. H., \& Burkert, A. 2010, ApJ, 725,2312

Peng, E. W., Ford, H. C., \& Freeman, K. C. 2004, ApJ, 602, 685

Peng, E. W., et al. 2006, ApJ, 639, 95

Peng, E. W., et al. 2008, ApJ, 681, 197

Press, W. H., Teukolsky, S. A., Vetterling, W. T., \& Flannery, B. P. 2007, Numerical Recipes 3rd Edition: The Art of Scientific Computing (New York: Cambridge: Univ. Press)

Proctor, R. N., Forbes, D. A., Romanowsky, A. J., Brodie, J. P., Strader, J., Spolaor, M., Mendel, J. T., \& Spitler, L. 2009, MNRAS, 398, 91

Pu, S. B., Saglia, R. P., Fabricius, M. H., Thomas, J., Bender, R., \& Han, Z. 2010, A\&A, 516, A4

Puzia, T. H., et al. 2004, A\&A, 415, 123

Qu, Y., Di Matteo, P., Lehnert, M., van Driel, W., \& Jog, C. J. 2010, A\&A, 515, A11

Romanowsky, A. J., Strader, J., Spitler, L. R., Johnson, R., Brodie, J. P., Forbes, D. A., \& Ponman, T. 2009, AJ, 137, 4956

Scorza, C., \& Bender, R. 1995, A\&A, 293, 20

Searle, L., \& Zinn, R. 1978, ApJ, 225, 357

van Dokkum, P. G., et al. 2008, ApJ, 677, L5

Vitvitska, M., Klypin, A. A., Kravtsov, A. V., Wechsler, R. H., Primack, J. R., \& Bullock, J. S. 2002, ApJ, 581, 799

White, S. D. M. 1980, MNRAS, 191, 1P

Wuyts, S., Cox, T. J., Hayward, C. C., Franx, M., Hernquist, L., Hopkins, P. F., Jonsson, P., \& van Dokkum, P. G. 2010, ApJ, 722, 1666

Zolotov, A., Willman, B., Brooks, A. M., Governato, F., Brook, C. B., Hogg, D. W., Quinn, T., \& Stinson, G. 2009, ApJ, 702, 1058 